\title{
Can Electrons Act as Antioxidants? A Review and Commentary
}

\author{
JAMES L. OSCHMAN, Ph.D.
}

\begin{abstract}
A previous study demonstrated that connecting the human body to the earth during sleep (earthing) normalizes the daily cortisol rhythm and improves sleep. A variety of other benefits were reported, including reductions in pain and inflammation. Subsequent studies have confirmed these earlier findings and documented virtually immediate physiologic and clinical effects of grounding or earthing the body. It is well established, though not widely known, that the surface of the earth possesses a limitless and continuously renewed supply of free or mobile electrons as a consequence of a global atmospheric electron circuit. Wearing shoes with insulating soles and/or sleeping in beds that are isolated from the electrical ground plane of the earth have disconnected most people from the earth's electrical rhythms and free electrons. The most reasonable hypothesis to explain the beneficial effects of earthing is that a direct earth connection enables both diurnal electrical rhythms and free electrons to flow from the earth to the body. It is proposed that the earth's diurnal electrical rhythms set the biological clocks for hormones that regulate sleep and activity. It is also suggested that free electrons from the earth neutralize the positively charged free radicals that are the hallmark of chronic inflammation. A relationship between cortisol and inflammation was established in the pioneering work of $\mathrm{H}$. Selye published in the 1950s. Current biomedical research has led to an inflammation hypothesis that is establishing chronic inflammation as the culprit behind almost every modern chronic illness. The research summarized here and in subsequent reports provides a basis for a number of earthing technologies that restore and maintain natural electrical contact between the human body and the earth throughout the day and night in situations where going barefoot on the earth is impractical. It is proposed that free or mobile electrons from the earth can resolve chronic inflammation by serving as natural antioxidants.
\end{abstract}

\section{INTRODUCTION}

A previous report by Ghaly and Teplitz ${ }^{1}$ demonstrated that connecting the human body to the earth during sleep normalizes the daily rhythm of cortisol, widely recognized as the "stress hormone." The researchers also noted improvements in sleep, reduction in pain and inflammation, and other beneficial effects. These findings confirmed earlier pilot studies of Ober $^{2}$ and Ober and Coghill. ${ }^{3}$ The method involved the use of an electrically conductive mattress pad. Conductive carbon or silver fibers woven into the pad coupled to a wire that connected to a foot-long metal rod inserted into the earth (Fig. 1).

In this paper, we refer to various methods of connecting the body with the earth with the term, "earthing." We distinguish this process from "grounding" as is used in electrical distribution systems, the electrostatic discharge industry, and lightning rods. Such grounding systems are designed to conduct large currents or voltages to the earth to protect equipment and its users. The personal earthing systems described here have a series resistor of 1 million ohms or greater that limits current flow to and from the body. Measurements

Nature's Own Research Association, Dover, NH.

Preparation of the manuscript was aided in part by funding from Barefoot Sales Corporation, and the author acknowledges a financial interest in this company. 


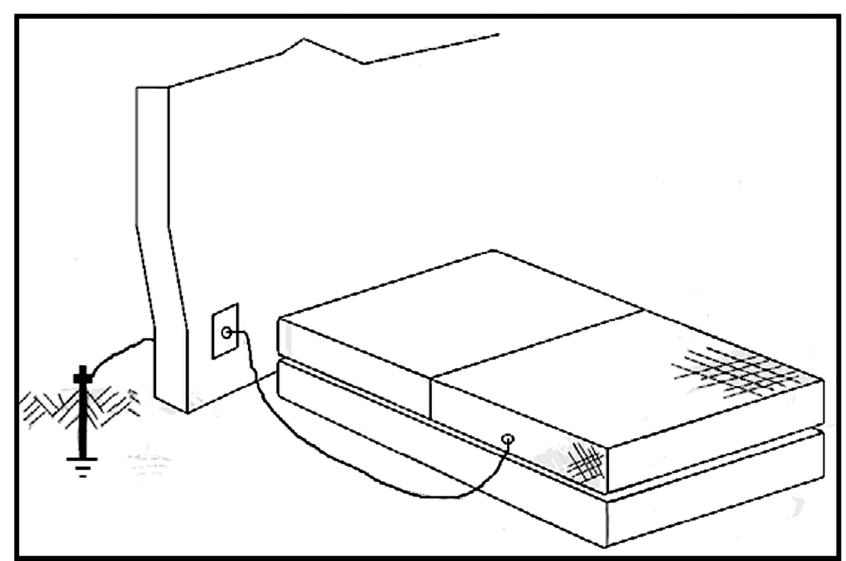

FIG. 1. Earthing sleep system. The mattress at the foot of the bed is covered with a pad containing conductive fibers (carbon or silver). Conductive fibers are electrically connected to the earth via a grounding rod.

of the voltage drop across this resistor provide estimates of the current flowing from the earth to the body and vice versa, and this is typically in the range of $1 \mu \mathrm{A}$ or less. ${ }^{4}$

The conductivity of the earth is variable and depends on soil moisture levels, mineral content, proximity to bodies of water, atmospheric conditions, and other factors. In spite of such variations, it is possible to demonstrate that a good earthing connection has been established by using a voltmeter to determine the degree to which the earthing system reduces induced currents on the body. The method is thoroughly described in the report of Ghaly and Teplitz. ${ }^{1}$ Local variations in the conductivity of the earth are probably not physiologically significant, given the enormous size of the earth and the tiny currents needed to earth the body, as described in the previous paragraph.

\section{THE CORTISOL RHYTHM}

A plausible explanation for the normalization of the cortisol rhythm documented by Ghaly and Teplitz is that the known diurnal rhythm in the earth's electrical field ${ }^{5}$ serves as the Zeitgebar or "time setter" of the cortisol rhythm in the body. If this is correct, it is logical that disconnection from the electrical field of the earth, as by wearing shoes with insulating soles, creates a situation in the body that is disruptive to the diurnal cortisol rhythm and that thereby interferes with normal sleep patterns. The wearing of shoes with rubber or plastic soles is a relatively recent development; throughout most of our evolutionary history humans have walked and slept directly on the earth or have used natural materials such as leather and straw for comfort. These natural materials, when combined with natural perspiration from the body, are adequately conductive to allow the passage of electrons from the earth to the body.
Since the pioneering work of Selye, ${ }^{6}$ cortisol has been widely regarded as the stress hormone ${ }^{7}$ and as a key factor in the body's defenses against disease. In thousands of experiments on laboratory animals, Selye demonstrated that prolonged stress activates the body's defense mechanisms, which Selye referred to as the alarm reaction. This reaction is followed by a resistance stage, during which defense reactions are maximized, and a final stage of exhaustion, in which defenses diminish and disappear. Selye referred to this three-step response-alarm, resistance, and exhaustion - as the "general adaptation syndrome." Importantly, he noted that during the course of the process, pathologic changes take place that are indistinguishable from the various diseases such as hypertension, myocardial infarction, nephrosclerosis, peptic ulcers, and rheumatoid arthritis, which Selye referred to as diseases of adaptation.

Hence, the findings of Ghaly and Teplitz tie in with the early findings of Selye regarding the role of cortisol in adaptation to stress. It has also been documented that disruption of circadian cortisol rhythms can contribute to a multitude of adverse health conditions, including sleep disorders, hypertension, cardiovascular disease, stroke, decreased bone density, decreased immune response, mood disturbances, autoimmune disease, and abnormal glucose levels. ${ }^{8}$ Cortisol has been implicated in the regulation of sleep, ${ }^{9}$ and $24-$ hour hypersecretion of cortisol has been linked to chronic insomnia. ${ }^{10,11}$ Excess cortisol has also been connected with inflammatory pain, pain perception, ${ }^{3,12}$ and depression. ${ }^{13}$

This and subsequent reports summarize evidence that connecting the human body to the earth has an additional role in the stress response by allowing free (mobile) electrons from the earth to enter the body where they act as natural antioxidants. This finding relates to the diseases of adaptation described by Selye as well as to the recent medical research implicating chronic inflammation with almost every modern disease.

\section{PHYSIOLOGIC CHANGES WITH EARTHING}

In 2006 Chevalier, Mori and Oschman published a double-blinded study documenting the effects of earthing on 58 healthy adult subjects. Earthing produced statistically significant and nearly instantaneous reductions in overall stress levels and tensions in the body as measured by electroencephalograms, electromyograms, and blood volume pulse. Taken together, the physiologic changes indicate reductions in overall stress levels and tensions, and a shift in autonomic balance upon earthing. ${ }^{14}$ As an example, Figure 2 shows changes in trapezius muscle tension in two subjects, measured with electromyography. At the moment the earthing lead is connected, there is a virtually instantaneous normalization in tension. Muscles that are tense relax, and muscles that are hypotoned develop normal tension. The hypertoned muscle (upper trace in Fig. 2) was obtained from an indi- 


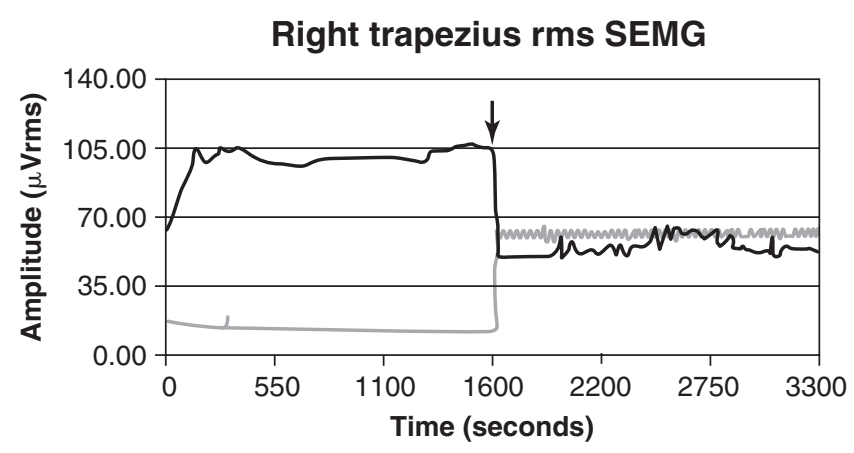

FIG. 2. Changes in trapezius electromyogram recordings with earthing patch connected (arrow). The hypertoned muscle (upper trace) was monitored on an individual experiencing acute pain from an injury the day before the test. The hypotoned muscle (lower trace) was from an individual with normal fatigue. SEMG, surface electromyogram.

vidual experiencing acute pain from an injury the day before the test, and the hypotoned muscle (lower trace) was obtained from an individual with normal fatigue.

\section{HEALING AND RECOVERY}

The effectiveness of connecting the body to the earth for enhancement of performance, facilitating more rapid recovery and the reduction of injury repair time was observed by Dr. Jeffrey Spencer during the most recent three Tour de France victories of the US Cycle Team lead by Lance Armstrong. Dr. Spencer's obligation during the Tour was to maintain the health and optimum performance of the team. The Tour was not a scientific expedition, and there was no time to document thoroughly the effects of the therapeutic tools that were used during what is arguably the most physically demanding athletic event in the world. However, Dr. Spencer has specialized in working with elite athletes functioning at the cutting edge of performance. He worked with the cycle team for the first 4 years of the Tour, before utilizing earthing techniques. The following observations are offered in the context of Dr. Spencer's experience and expertise. In contrast to the first 4 years of working with the cycle team during the Tour, Spencer noted that the use of the earthing techniques correlated with:

- Reduction of pain

- Less pain medication

- Better sleep

- Faster healing

- Less sickness

- Better oral health

- Better ability to handle stress

- More stable psychologic disposition

- More energy

- Feeling more rested

- Improved range of motion
- Less tension

- Less scarring

- Greater strength and endurance

- No jet lag

- Less stiffness and soreness.

Dr. Spencer also carried out preliminary studies using medical infrared imaging. He was able to demonstrate that connecting the body to the earth facilitated rapid reduction of inflammation. These unpublished findings have been confirmed, as described next.

\section{CLINICAL TRIALS}

Two clinical trials used medical infrared imaging and clinical outcomes to assess changes taking place when the body was connected to the earth (additional cases are described in Appendix I).

Inflammation is the earliest stage of almost all major health challenges. Because it measures heat, medical infrared imaging easily detects acute or chronic inflammatory conditions. The value of the technique has been documented by countless research studies. ${ }^{15}$ The method can measure changes as small as 1/100th of a degree C, providing "biomarkers, biofingerprints" or "risk indicators" that reveal the beginnings of tumors, toxic accumulations, and disease, months or even years earlier than other imaging procedures. In spite of the great accuracy, to 1/100th of a degree C, experts who use medical infrared imaging are less interested in the absolute temperature of a part of the body than they are in locating "hot spots" and left-right imbalances that correspond to areas of discomfort and that are indicative of inflammation. In some cases, the temperature scale (color bar) had to be shifted to encompass the warmer thermal profiles after earthing. See the legends for each illustration for details of the most important observations.

Figure 3 shows medical infrared imaging of a 65 -yearold woman with chronic thigh and knee pain on the right side, ankle and foot pain, and swelling of the left foot. The pain in her legs interfered with sleep; she woke stiff and sore and was tired all day. Prolonged medical treatment had not helped. After using the earthing sleep system (Fig. 1) for 4 nights, she reported $91 \%$ reduction in pain, $50 \%$ improvement in restful sleep, $50 \%$ reduction in insomnia, $50 \%$ reduction in sleepiness during the day, $81 \%$ reduction in pain interfering with sleep, $50 \%$ reduction in leg achiness during sleep, and 50\% reduction in waking stiff and sore. The patient reported steady continued improvement at a 40-day follow-up. Similar results were obtained with dozens of other patients (Appendix I).

Infrared images taken before use of the earthing system (at the top in Fig. 3) show the most significant areas of inflammation (arrows) that corresponded precisely with her areas of complaint. The bottom images, taken after 4 nights 
of sleeping on the earthing sleep system, show significant reduction in inflammation and restoration of normal thermal symmetry.

A second clinical study employed the earthing patch shown in Figure 4. The patch includes a peel-away strip over a conductive adhesive layer and can be applied to any area of the body. As with the earthing sleep system, the patch connects to a grounding rod in the earth. More than 20 patients were studied; all showed immediate and permanent reductions in pain, improved overall health and return to a normal lifestyle. Conditions treated included chronic myofascial pain syndrome, muscular strains, ligamentous sprains, peripheral neuropathies, carpal tunnel syndrome, inflammatory joint conditions, Lyme disease, and chronic sinusitis. Within 2-4 weeks (receiving half-hour treatments 2-3 times per week), up to $80 \%$ improvement took place in over $60 \%$ of the cases.

Figure 5 shows results with a 33-year-old woman who had a gymnastics injury at the age of 15 . She had an 18year history of chronic right knee pain, tenderness, swelling, and instability. She was unable to stand for long periods and simple actions such as driving increased the symptoms. She had been undergoing medical treatment and physical therapy on and off for many years with minimal results. Figure 5 shows infrared images (walking position, with views of the insides of both knees) before and after using the earthing patch. The arrow points to the exact location of the patient's pain and inflammation. Note the considerable reduction in inflammation in the bottom images taken after only 30 minutes of earthing treatment. Over a 12-week period, she continued to improve to the point that she was able to compete in a half-marathon.

Another patient had an acute right ankle sprain with significant swelling. A 30-minute treatment with the earthing patch significantly reduced the heat radiating from the ankle and improved circulation to the foot and toes. There was a rapid "quieting" of the sympathetic nervous system, and a rapid reduction in the acute phase of the inflammatory process.

These observations and others like them described in Appendix I reveal extremely rapid resolution of both acute and chronic inflammation and its consequences using earthing techniques. In view of recent research identifying low-level chronic inflammation (also called "silent inflammation") ${ }^{16}$ as the underlying cause of a wide range of diseases and disorders not previously thought of as inflammatory, the above findings are highly significant.

Finally, note in particular that after the first subject described in Appendix I used the earthing sleep system, her hands and feet appear warmer than the arms and legs, respectively (Figs. A-1 and A-2, in Appendix I). This is explained by a phenomenon called "distal vasodilation." ${ }^{17}$ The distal skin regions of the body (hands and feet) are the major sites for vasomotor heat loss, whereas the proximal skin regions (arms, trunk, and legs) play a lesser role in thermoregulation. ${ }^{18}$ Distal vasodilation involves arteriovenous anastomoses that are particularly abundant in the hands and feet ${ }^{19}$ and can shunt blood directly from arterioles to veins. According to Guyton, ${ }^{20}$ "the rate of blood flow into this venous plexus can vary tremendously-from barely above zero to as great as 30 per cent of the total cardiac output. A high rate of blood flow causes heat to be conducted from the internal portions of the body to the skin with great efficiency ...." At the same time, the glabrous (hairless) epidermis of both hands and feet is rich in eccrine sweat glands that can provide for localized sweating. ${ }^{21}$ While sweat glands are distributed over the entire body surface, they are particularly abundant on the palms of the hands and soles of the feet. Under appropriate conditions, the sympathetic nervous system can increase circulation to the skin of the hands and feet and activate the sweat glands, increasing radiative and evaporative cooling from the hands and feet. The improved circulation to the hands and feet documented by Figures A1 and A-2 (in Appendix I) may correlate with the improvements in sleep noted by the subject in Case Study \#1. Specifically, Kräuchi and colleagues ${ }^{17}$ have demonstrated that the distal-to-proximal skin temperature gradient is the best predictor variable for sleep-onset latency (compared with core body temperature or its rate of change, heart rate, melatonin onset, and subjective sleepiness ratings). Their study provides evidence that selective vasodilation of distal skin regions (and hence heat loss) promotes the rapid onset of sleep.

\section{THE INFLAMMATION HYPOTHESIS}

Study of the relationship between chronic inflammation and disease has attracted the attention of investigators around the world. The extent of this interest is documented in Table 1, which shows the results of a PubMed search for studies of various diseases combined with inflammation as a second key word. The 14 categories searched resulted in over 70,000 citations, with the largest number being citations for cancer. A relationship between cancer and inflammation due to chronic infection has been suspected, but not proven, for many years. In a 1986 study, for example, one researcher compared the inflammatory response to the wound healing response, saying tumors were wounds that do not heal.

Research connecting inflammation with chronic disease was given a dramatic impetus as a result of a series of studies by Ridker and colleagues at Harvard Medical School. Suspecting that inflammation is involved in the pathogenesis of cardiovascular events, these researchers measured the levels of inflammatory markers in a prospective controlled study among 28,263 apparently healthy postmenopausal women over a mean follow-up period of 3 years. They assessed the risk of cardiovascular events associated with a variety of established inflammatory markers. Of 12 markers studied, high-sensitivity C-reactive protein (hs-CRP) proved to be the best risk predictor for cardiovascular events. ${ }^{22}$ A group in Taipei, Taiwan confirmed these results in a study published in $2004 .{ }^{23}$ 
FIG. 3. Medical infrared images of a 65-year-old woman with chronic thigh and knee pain on the right side, ankle and foot pain, and swelling of the left foot. Top row images show lower extremities taken before using the earthing sleep system. Arrows denote most significant areas of inflammation and correspond precisely with subject's areas of complaint. Bottom images taken after 4 nights sleeping on earthing sleep system. Note considerable reduction in inflammation and return toward normal thermal symmetry. Patient reported steady continued improvement at a 40-day follow-up.

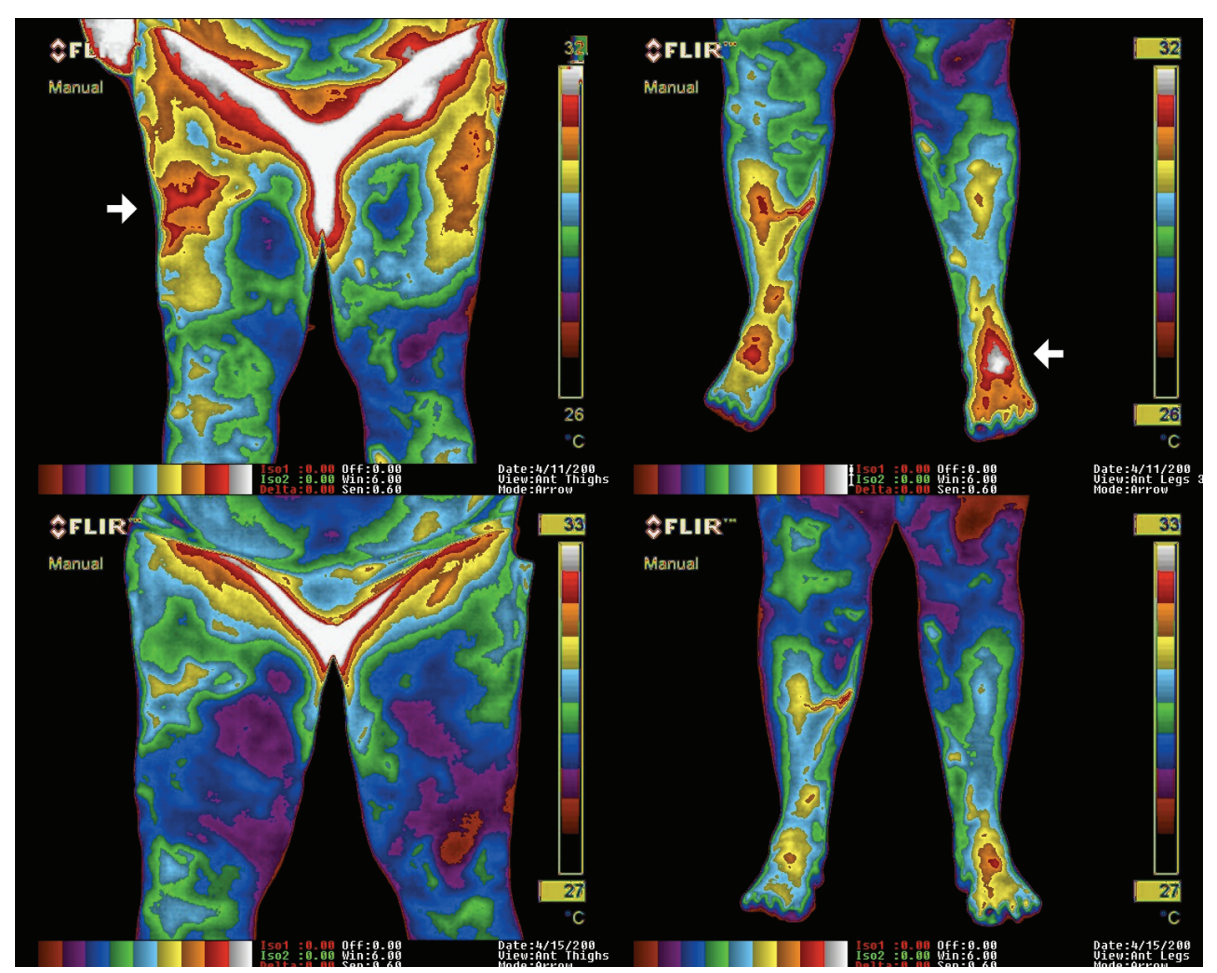

FIG. 4. Clinical earthing patch system. Patch includes a peel-away strip over a conductive adhesive layer, and can be applied to any area of the body. As with the earthing sleep system, the patch is connected with the earth via a grounding rod.
FIG. 5. Medical infrared imaging results with a 33-year-old woman who had a gymnastics injury at the age of 15 . She had an 18-year history of chronic right knee pain, swelling, and instability. Top row of images taken in walking position to show inside of both knees. Arrow points to exact location of patient's pain and shows significant inflammation. Lower images were taken after 30 minutes of exposure to clinical earthing using patch system shown in Figure 4. Note significant reduction of inflammation in knee area. After 6 days of clinical earthing, patient reported a $50 \%$ reduction in pain. After 4 weeks of treatment, patient was able to play soccer, and by 12 weeks she went waterskiing.

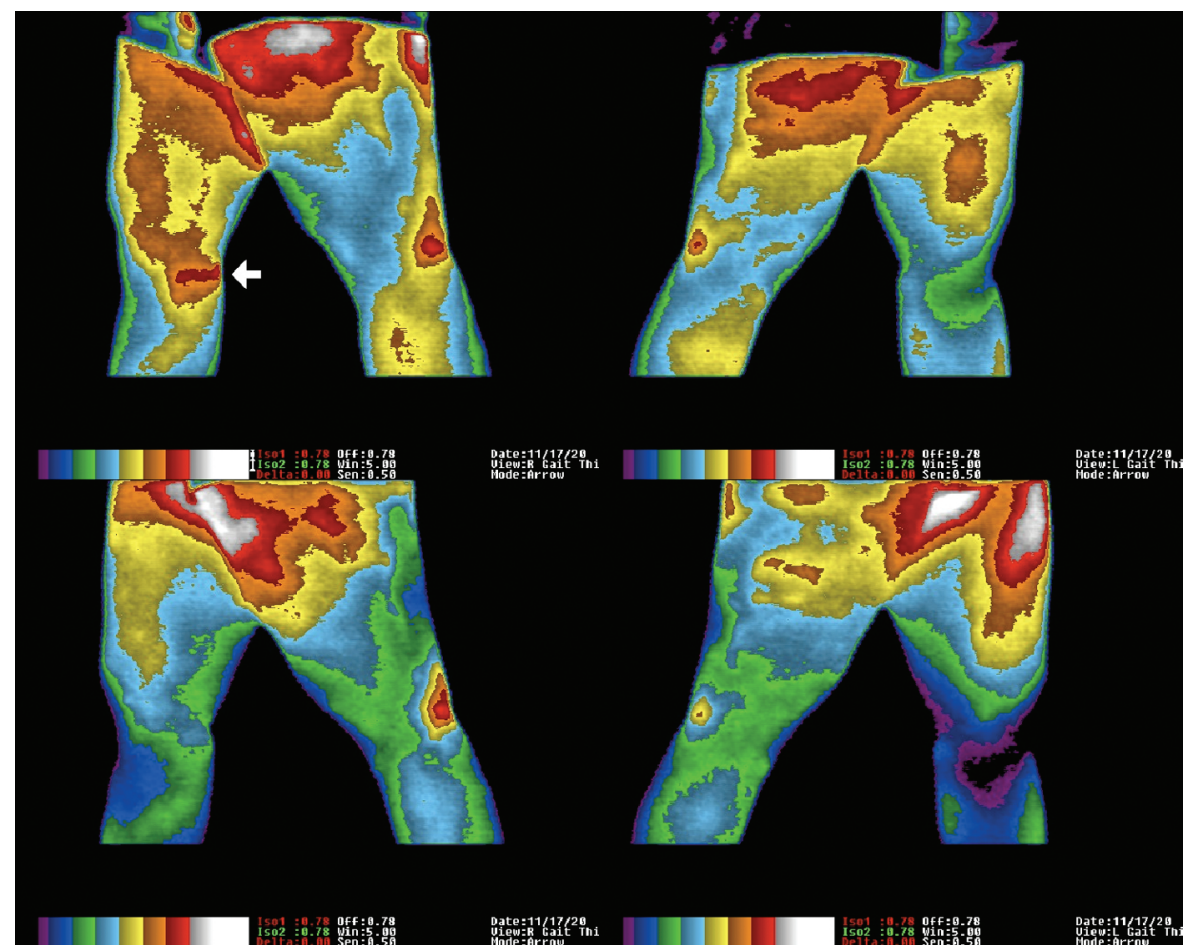


In 2001, Ridker and colleagues studied the risk factors for systemic atherosclerosis in 14,916 initially healthy U.S. male physicians. Total high-density lipoprotein cholesterol ratio and hs-CRP were the strongest independent predictors of development of peripheral arterial disease. ${ }^{24}$

In 2002, Ridker and colleagues reported measurements of hs-CRP and low-density lipoprotein cholesterol in 27,939 apparently healthy American women who were then followed for a mean of 8 years for the occurrence of myocardial infarction, ischemic stroke, coronary revascularization, or death from cardiovascular causes. They found that baseline levels of each marker had a strong linear relation with the incidence of cardiovascular events. Screening for both biologic markers provided better prognostic information than screening for either alone. ${ }^{25}$

Another study from the Harvard group published in 2002 examined the relationship between use of hormone replacement therapy and coronary heart disease among 75,343 women. Hormone replacement therapy was associated with significantly elevated median hs-CRP levels and it increased the odds ratio for coronary heart disease. ${ }^{26}$ Further study by Ridker and colleagues revealed a correlation between chronic inflammation and sudden cardiac death. ${ }^{27}$

As a result of these studies, and many others like them, the American Heart Association and the Centers for Disease Control and Prevention recommended in 2003 that doctors include a test for free radicals in their medical check-ups to determine a patient's risk for heart disease. ${ }^{28}$ Subsequently there has been a veritable explosion of research into the association of inflammation and inflammatory markers with a wide range of chronic illnesses. Today, nearly every branch of medicine and surgery includes the study of inflammation. ${ }^{29}$ Inflammation is now thought to be the underlying mechanism of more than 80 chronic illnesses involving almost every human organ system, including diseases of the nervous, gastrointestinal, endocrine, and respiratory systems as well as the skin and connective tissues. In all of these diseases, the underlying problem is similar-the body's immune system is causing harm to the organs it was designed to protect.

Ongoing research has confirmed a role for inflammation in aging, ${ }^{30}$ Alzheimer's disease, ${ }^{31}$ asthma, ${ }^{32}$ atherosclerosis, ${ }^{33}$ bowel disorder, ${ }^{34}$ cancer, ${ }^{35}$ cystic fibrosis, ${ }^{36}$ diabetes, ${ }^{37}$ meningitis, ${ }^{38}$ multiple sclerosis, ${ }^{39}$ osteoporosis, ${ }^{40}$ prostate cancer, ${ }^{41}$ psoriasis, ${ }^{42}$ and rheumatoid arthritis. ${ }^{43}$ The above references are examples from the 2006 literature alone-an indication that this is now one of the most active areas in clinical research.

\section{THE INFLAMMATORY RESPONSE}

Inflammation is defined as "a localized protective response in trauma or microbial invasion that destroys, dilutes, or walls-off the injurious agent and the injured tissue." Acute inflammation is characterized by the classic signs of pain, heat, redness, swelling, and loss of function. Microscopically, it involves a complex series of events, including dilation of arterioles, capillaries, and venules, with increased permeability and blood flow; exudation of fluids, including plasma proteins; leukocyte migration into the inflammatory focus; and secretion of free radicals into the area. ${ }^{44}$

Part of the inflammatory response involves immune cells, including neutrophils and various types of phagocytes that secrete powerful oxidizing agents (free radicals) in a process known as the respiratory burst. A complex mix of reactive molecules such as hydrogen peroxide, oxidized halogens, chloramines, and oxidizing radicals such as hydroxyl radi$\mathrm{cal},{ }^{\circ} \mathrm{OH}$, accumulate at the site of inflammation where they aid in the destruction of invading microorganisms and tissue debris. ${ }^{45}$ To restore their electrical neutrality, these agents tear electrons from the structures of invading organisms and damaged cells, rapidly destroying them.

Ideally, the inflammatory process winds down when all of the damaged cells and bacteria have been cleared away. Sometimes, however, for reasons that are the subject of current research, as well as the factors discussed in this paper, the inflammatory process does not fully resolve. Some free radicals may diffuse into regions adjacent to the original site of injury or infection and begin to damage healthy cells. Cells have some capacity to regenerate and recover from free radical damage and there are enzymes that can repair broken strands of DNA. However, if the process continues long enough, the repair processes are unable to keep up and cells become damaged beyond repair. Additional signals are sent to the immune system, which responds by delivering more neutrophils and free radicals. The result is a vicious cycle of low-level inflammation that can eventually compromise important functions and lead to chronic disease, including disorders of organs distant from the original trauma.

The inflammatory process is taking place to some degree within every human body. It is a continuing struggle to main-

Table 1. PubMed Citations for Diseases Searched with inflammation as a SECONd SEARCh Term, as OF SEPTEMBER 12, 2007

- Aging: 2098

- Alzheimer's disease: 1238

- Asthma: 9135

- Atherosclerosis: 4578

- Bowel disorders: 14,723

- Cancer: 24,170

- Cystic fibrosis: 1338

- Diabetes: 6102

- Meningitis: 4812

- Multiple sclerosis: 1974

- Osteoporosis: 503

- Prostate cancer: 514

- Psoriasis: 1402

- Rheumatoid arthritis: 7087

Total: 79,854 citations 
tain normal functioning and survival in the face of small and large insults. The swelling and inflammation (cut, boil, sprain, abscess, or bruise) are intelligent responses of the body. Often they constitute a sort of quarantine, a barricade of inflammatory tissue that prevents microbes or poisons from spreading further throughout the body.

\section{CORTISOL AND THE INFLAMMATORY POUCH}

In 1950, Reichstein, Kendall, and Hench were jointly awarded the Nobel Prize for Physiology or Medicine for their work on the hormones of the adrenal cortex. Their efforts culminated in the isolation of cortisone and the discovery of its therapeutic value in the treatment of rheumatoid arthritis. ${ }^{46}$ A few years later, the famous stress researcher, Hans Selye, discovered that it was possible to inject air under the skin of a rat and cause the formation of a walled-off sack. ${ }^{6}$ These "inflammatory pouches" provided Selye and others with a reproducible model for studying the inflammatory process and the ways it is influenced by antiinflammatory corticoid hormones.

When Selye injected microbes or corrosive chemicals into these air sacks, the tissue lining the sacs developed inflammatory barricades that protected the adjacent tissues. However, when rats were pretreated with corticoids, the inflammatory barricade did not form. Instead, the irritants introduced into the air sac spread into adjacent tissues. This led to the conclusion that the so-called anti-inflammatory hormones act nonspecifically by inhibiting the immediate inflammatory defense reactions to toxic agents. Selye concluded that this was advantageous when the irritant was mild and unlikely to cause much damage, but could be detrimental if the irritant was severe and could damage surrounding tissues.

In his later writings, Selye referred to "syntoxic" and "catatoxic" responses. Syntoxic stimuli tone down inflammatory reactions through the release of corticoid hormones. Under these circumstances, the invading stressor is relatively innocuous and is easily tolerated (e.g., the mild inflammation experienced as a disease symptom or allergy). In contrast, catatoxic stimuli lead to the production of destructive enzymes that attack the pathogen and degrade it. ${ }^{47}$

\section{FOCAL INFECTION AND DISEASE}

A focal infection is a circumscribed area that may or may not give rise to clinical manifestations. During the 17th century, it was discovered that dental infections could produce a wide variety of ailments throughout the body that could be treated by removing the infected teeth. In the 20th century, American physicians became aware that focal infections could produce symptoms in other parts of the body with or without the transfer of pathogens via the blood. A variety of theories arose to explain how this could happen.
In his book, The Stress of Life, Selye discussed the relationship between focal infection and chronic or systemic disease. He favored the logical explanation that the architecture of the inflammatory barricade might be a key factor. He conceived conditions where poisons leaked slowly into the circulatory system, creating problems such as heart disease or nephritis in organs distant from the focus of the infection. Selye performed experiments showing that an infection in one part of the body could produce inflammation of the heart valves, as often happens in children suffering from rheumatic fever.

Focal infection has continued to be a valuable concept in dentistry. In 1951, the problem of focal infection was discussed at length in the Journal of the American Dental Association. ${ }^{48}$ Gammal $^{49}$ lists 389 MEDLINE $^{\circledR}$ references to focal infection in relation to dentistry between 1960 and 1998. PubMed lists 3848 references to focal infection, with more than 100 published in 2007. To summarize this research: Necrotic tissue breakdown products in a small inflammatory pouch can leak into the blood and lymphatic circulation, producing a slow but progressive atrophy in various organs a distance from the original site of trauma. Barnett provides an upto-date review of this topic. ${ }^{50}$ This progressive organ atrophy, then, is the most probable link between focal chronic inflammation and the various chronic diseases listed earlier.

\section{ELECTRONS AS ANTIOXIDANTS}

The inflammation theory, which has attracted considerable interest among medical researchers in recent years, connects chronic disease with a situation that is describable in electronic or energetic terms. A free radical is a molecule that is missing an electron. Its destructive effects are explained in terms of the rapid and violent reactions taking place as electrical charges are redistributed between the reacting molecules. The "violent reactions" are the breaking of chemical bonds responsible for the integrity of the cell walls of bacteria, cell membranes, DNA, damaged connective tissue, and other structures. We observe this violent reaction when we watch a fire, since burning is the process by which oxygen tears electrons from the bonds holding matter together, liberating heat and light. Oxygen is the ultimate "oxidizing agent" and the electron is the ultimate "reducing agent." We are all familiar with a similar but somewhat slower "burning" process that takes place when we experience the heat, redness, swelling, pain, and loss of range of motion that can accompany an inflammatory condition. Note that we have qualified the previous statement by saying that these symptoms can accompany an inflammatory condition. Experience with the earthing techniques described here has shown that these characteristic inflammatory conditions may be reduced or even prevented if the body is connected to the earth immediately after an injury. Dr. Spencer repeatedly confirmed this in his work with athletes during the Tour de France. 
Since inflammation is a consequence of deficiency in negative charge, any mechanism that delivers electrons to a site of injury will lessen the likelihood of a persistent vicious cycle of inflammation. Hence, medical research is indicating that people who regularly take drugs to reduce levels of inflammatory markers tend to experience fewer of the chronic diseases and disorders associated with aging. ${ }^{51}$ Consequently many people add to their diets dark chocolate, ${ }^{52}$ turmeric, ${ }^{53,54}$ bamboo, ${ }^{55}$ and other nutritional supplements that are rich in free-radical scavengers. Anti-inflammatory drugs and antioxidants are electrically charged molecules that carry excess electrons to sites of inflammation where they reduce free radicals. Our hypothesis is that free or mobile electrons can act directly on free radicals, and that this happens naturally when the body is in contact with the earth, which is a natural source of free electrons.

Anti-inflammatory molecules can become free radicals themselves, once they have donated electrons to neutralize free radicals. Moreover, metabolic processes must remove the antioxidant that has given up its electron. This poses additional demands on the energy system of the organism. In contrast, free electrons act directly on free radicals and do not have the disadvantages of chemical antioxidants. It therefore appears that free (mobile) electrons from the earth can serve a simple and direct "anti-inflammatory" role by neutralizing free radicals.

\section{THE EARTH AS A SOURCE OF ELECTRONS}

It is well established, though not widely appreciated, that the surface of the earth possesses a limitless and continuously renewed supply of free or mobile electrons. The earth's surface is electrically conductive and is maintained at a negative potential by an atmospheric electrical circuit. ${ }^{56,57}$ There are three main generators in the global electric circuit: the solar wind entering the magnetosphere, the ionospheric wind, and meteorological activities. ${ }^{56,58}$

The conductivity of the earth varies somewhat from place to place, depending on water and mineral content, the level of the water table, vegetation, and other factors. However, these factors have relatively little effect on the ability of an earth connection to allow electrons to flow from the earth to the body and vice versa. This can be stated with certainty because of previous measurements. ${ }^{1-4}$

\section{CONDUCTION OF FREE (MOBILE) ELECTRONS WITHIN THE BODY}

The results of various investigations summarized here show that there are rapid and profound anti-inflammatory effects from restoring and maintaining a natural electrical contact between the earth and the human body. The hypothesis presented proposes that these anti-inflammatory ef- fects are due to the flow of free electrons from the earth to the body, which is achieved by standing barefoot on the earth, by use of the earthing sleep system shown in Figure 1 , or via clinical applications using the earthing patch shown in Figure 3. This hypothesis raises the question of how electrons can be conducted from the skin surface to pockets of inflammation in various tissues.

Electrically conducting pathways between the skin surface and internal tissues and organs are utilized in a variety of diagnostic tools such as the electrocardiogram and electroencephalogram. These techniques utilize electrodes on the skin surface to detect electrical fields generated by the heart and brain, respectively. Obviously, if there is a conduction path from an internal organ to the skin surface, the circuit will work in the reverse direction, from the skin to the organ. Conduction from the skin to the interior of the body is also demonstrated by the success of various therapies utilizing microcurrent* and frequency-specific microcurrent. ${ }^{59}$ Additional documentation comes from extensive research on acupuncture and electroacupuncture. ${ }^{60}$ Electrical aspects of organ function measured at acupuncture points on the skin surface can be used for diagnosis; electrical signals introduced into the same skin points can enhance functions of corresponding organs. ${ }^{61}$

It is generally accepted that dissolved electrolytes are the charge carriers in living systems. It is also assumed that the body is a "volume conductor," meaning that the skin houses a homogeneous solution of electrolytes and proteins. ${ }^{62} \mathrm{How}-$ ever, the volume conductor assumption disregards some significant areas of biomedicine: anatomy, histology, cell biology, and membrane biology. Moreover, the physics of earthing makes it extremely unlikely that the electrons from the earth are causing ion fluxes to sites of inflammation. Finally, sites of inflammation can be walled-off in inflammatory pouches, as originally described by Selye. This fact argues against using the volume conductor assumption when considering the access of anti-inflammatory molecules or electrons to pockets of free radicals.

Significant concentrations of negatively charged ions, particularly chloride, are present virtually everywhere in body fluids; these ions are obviously not acting as antioxidants. Even if electrolytes were somehow acting as antioxidants, we would have to explain how contact with the earth increases their availability at sites of inflammation. It is highly unlikely that the ubiquitous electrolytes, with their large hydration shells, can act as antioxidants by neutralizing free radicals.

The phenomena described in this paper raise questions about the very nature of the inflammatory response and the role of free or mobile electrons in physiologic processes. These issues will be discussed further in subsequent publications ${ }^{63}$ (Oschman, unpublished observations).

\footnotetext{
*The database for the National Library of Medicine lists 450 references to microcurrent therapy.
} 


\section{CONCLUSIONS}

A recent editorial in this journal ${ }^{64}$ pointed out that study of the earthing phenomenon sheds new light on the classical question raised by the noted biologist, T.H. Huxley: "The question of all questions for humanity, the problem which lies behind all others and is more interesting than any of them, is that of the determination of our place in nature and our relation to the cosmos. ${ }^{\prime 65}$ The research summarized here and in the other papers in this series indicates that there are profoundly therapeutic effects from restoring and maintaining, as much as is practical, a natural electrical contact between the body and the earth. Understanding the significance of this earth connection is a step in comprehending our place in nature.

The evidence suggests that free (mobile) electrons from the earth are natural antioxidants. This requires us to revise our thinking about the inflammatory process at a fundamental level. Specifically, Selye showed that different kinds of irritants produce different degrees of inflammatory responses (syntoxic and catatoxic), and that hormones from the adrenal cortex mediate and modulate these responses. The effects of earthing described here introduce another variable to the inflammation equation: the degree of conductive contact with the earth. Stated differently, loss of direct contact with the earth appears to introduce another kind of adaptive stress, an "electron deficiency," which compromises the immune system by slowing the resolution of inflammatory responses.

The Ghaly and Teplitz study showed that contact with the earth can influence cortisol rhythms. The results summarized here show another influence of earth contact: providing free (mobile) electrons. This has significance for a wide range of therapeutic modalities that introduce electricity into the body, or that may cause electrons to move about within the body. It is possible that the benefits of many bodywork, energetic and movement therapies, as well as of various energy medicine devices, are partly due to their ability to enable mobile electrons to penetrate into the inflammatory pockets where they neutralize the free radicals that contribute to so many different chronic diseases.

\section{ACKNOWLEDGMENTS}

Thanks go to Clinton C. Ober, inventor of the earthing systems described in this report, for many valuable discussions of his observations on thousands of people who have used the system he developed and patented. The author is indebted to Jeff Spencer, M.A., D.C., CCSP, for reporting the results of applying the earthing sleep system during four successful competitions of the U.S. Cycling Team in the Tour de France. The author thanks William Amalu, D.C., D.A.B.C.T., D.I.A.C.T., FIACT for conducting the clinical trials with medical infrared imaging and clinical outcome measures. Dr. Amalu is president of the International Acad- emy of Clinical Thermology. Valuable comments on the manuscript were provided by Nora Oschman, Dale Teplitz, M.A., and M.J. Pangman.

\section{REFERENCES}

1. Ghaly M, Teplitz D. The biological effects of grounding the human body during sleep, as measured by cortisol levels and subjective reporting of sleep, pain and stress. J Altern Complement Med 2004;10:767-776.

2. Ober AC. Grounding the human body to earth reduces chronic inflammation and related chronic pain. ESD J July 2003.

3. Ober AC, Coghill RW. Does grounding the human body to earth reduce chronic inflammation and related chronic pain? Presented at the European Bioelectromagnetics Association annual meeting, on December 12, 2003, Budapest, Hungary.

4. Applewhite R. Effectiveness of a conductive patch and a conductive bed pad in reducing induced human body voltage via the application of earth ground. Eur Biol Bioelectromagnetics 2005;1:23-40.

5. Feynman R, Leighton RB, Sands M. The Feynman Lectures on Physics. Volume II. Reading, MA: Addison-Wesley Publishing Company, 1964:9-2-3.

6. Selye H. The Stress of Life. New York: Mc-Graw-Hill Book Company, 1956.

7. Bjorntorp P. Do stress reactions cause abdominal obesity and comorbidities? Obes Rev 2001;2:73-86.

8. Alschuler L. Stress: Thief in the night. Int J Integ Med 2001; 3:27-34.

9. Follenius M, Brandenberger G, Bandesapt JJ, et al. Nocturnal cortisol release in relation to sleep structure. Sleep 1992; $15: 21-27$.

10. Vgontzas AN, Zoumakis M, Papanicolaou DA, et al. Chronic insomnia is associated with a shift of interleukin- 6 and tumor necrosis factor secretion from nighttime to daytime. Metabolism 2002;51:887-892.

11. Rodenbeck A, Huether G, Ruther E, Hajak G. Interactions between evening and nocturnal cortisol secretion and sleep parameters in patients with severe chronic primary insomnia. Neurosci Lett 2002;324:159-163.

12. Korszun A, Young EA, Singer K, et al. Basal circadian cortisol secretion in women with temporomandibular disorders. J Dent Res 2002;81:279-283.

13. Linkowski P, Mendlewicz J, Leclercq R, et al. The 24-hour profile of adrenocorticotropin and cortisol in major depressive illness. J Clin Endocrinol Metab 1985;61:429-438.

14. Chevalier G, Mori K, Oschman, JL. The effect of earthing (grounding) on human physiology. Eur Biol Bioelectromagnetics 2006;1:600-621.

15. Amalu WC, Hobbins WB, Head JF, Elliot RL. Infrared imaging of the breast-An overview. In: Bronzino JD, ed. The Biomedical Engineering Handbook, 3rd ed.: Medical Devices and Systems. Baton Rouge, LA: CRC Press, 2006:1-20.

16. Sears B. The Anti-Inflammation Zone: Reversing the Silent Epidemic That's Destroying Our Health. New York: Regan Books/Harper Collins, 2004.

17. Kräuchi K, Cajochen C, Werth E, Wirz-Justice A. Functional link between distal vasodilation and sleep-onset latency? Am 
J Physiol Regulatory Integrative Comp Physiol 2000;278: R741-R748.

18. Aschoff J. The extremities as effectors of physical temperature regulation [in German]. Wiener Med Wochenschr 1958;19/20:404-409.

19. Bergersen TK. A search for arteriovenous anastomoses in human skin using ultrasound Doppler. Acta Physiol Scand 1993; 147:195-201.

20. Guyton AC. Textbook of Medical Physiology. Philadelphia: W.B. Saunders, 1971:836.

21. Guyton AC. Textbook of Medical Physiology. Philadelphia: W.B. Saunders, 1971:834.

22. Ridker PM, Hennekens CH, Buring JE, Rifai N. C-reactive protein and other markers of inflammation in the prediction of cardiovascular disease in women. N Engl J Med 2000;342: 836-843.

23. Leu H-B, Lin C-P, Lin W-T, et al. Risk stratification and prognostic implication of plasma biomarkers in nondiabetic patients with stable coronary artery disease: The role of highsensitivity C-reactive protein. Chest 2004;126:1032-1039.

24. Ridker PM, Stampfer MJ, Rifai M. Novel risk factors for systematic atherosclerosis: A comparison of C-reactive protein, fibrinogen, homocysteine, lipoprotein(a), and standard cholesterol screening as predictors of peripheral arterial disease. JAMA 2001;285:2481-2485.

25. Ridker PM, Rifai N, Rose L, et al. Comparison of C-reactive protein and low-density lipoprotein cholesterol levels in the prediction of first cardiovascular events. N Engl J Med 2002; 347:1557-1565.

26. Pradhan AD, Manson JE, Rossouw JE, et al. Inflammatory biomarkers, hormone replacement therapy, and incident coronary heart disease: Prospective analysis from the Women's Health Initiative observational study. JAMA 2002;288:980 987.

27. Albert CM, Ma J, Rifai N, et al. Prospective study of C-reactive protein, homocysteine, and plasma lipid levels as predictors of sudden cardiac death. Circulation 2002;105:25952599.

28. Pearson TA, Mensah GA, Alexander RW, et al. Markers of inflammation and cardiovascular disease application to clinical and public health practice. A statement for healthcare professionals from the Centers for Disease Control and Prevention and the American Heart Association. Circulation 2003; 107:499-511.

29. Alenghat FJ, Ingber DE. Mechanotransduction: All signals point to cytoskeleton, matrix, and integrins. Science's STKE 2002 Online document at: http://stke.sciencemag.org/cgi/ content/full/OC_sigtrans;2002/119/pe6 Accessed February 12, 2002.

30. Alvarado C, Alvarez P, Puerto M, et al. Dietary supplementation with antioxidants improves functions and decreases oxidative stress of leukocytes from prematurely aging mice. Nutrition 2006;22:767-777.

31. Di Rosa M, Dell'Ombra N, Zambito AM, et al. Chitotriosidase and inflammatory mediator levels in Alzheimer's disease and cerebrovascular dementia. Eur J Neurosci 2006;23:26482656.

32. Joachim RA, Sagach V, Quarcoo D, et al. Upregulation of tumor necrosis factor-alpha by stress and substance $\mathrm{P}$ in a murine model of allergic airway inflammation. Neuroimmunomodulation 2006;12-13:43-50.

33. Folsom AR, Chambless LE, Ballantyne CM, et al. An assessment of incremental coronary risk prediction using C-reactive protein and other novel risk markers: The atherosclerosis risk in communities study. Arch Intern Med 2006;166: 1368-1373.

34. Zilberman L, Maharshak N, Arbel Y, et al. Correlated expression of high-sensitivity $\mathrm{C}$-reactive protein in relation to disease activity in inflammatory bowel disease: Lack of differences between crohn's disease and ulcerative colitis. Digestion 2006;73:205-209.

35. Allgayer H, Kruis W. From chronic inflammation to metastasizing colon cancer - the endless story of the NSAIDs. Z Gastroenterol 2006;44:611-613.

36. Clayton A, Knox AJ. COX-2: A link between airway inflammation and disordered chloride secretion in cystic fibrosis? Thorax 2006;61:552-553.

37. Ben-Mahmud BM, Chan WH, Abdulahad RM, et al. Clinical validation of a link between TNF-alpha and the glycosylation enzyme core 2 GlcNAc-T and the relationship of this link to diabetic retinopathy. Diabetologia 2006;49:2185-2191.

38. Keino H, Goto H, Mori H, et al. Association between severity of inflammation in CNS and development of sunset glow fundus in Vogt-Koyanagi-Harada disease. Am J Ophthalmol 2006;141:1140-1142.

39. Becher B, Bechmann I, Greter M. Antigen presentation in autoimmunity and CNS inflammation: How T lymphocytes recognize the brain. J Mol Med 2006;84:532-543.

40. Weitzmann MN, Pacifici R. Estrogen deficiency and bone loss: An inflammatory tale. Clin Invest 2006;116(5):1186-1194.

41. MacLennan GT, Eisenberg R, Fleshman RL, et al. The influence of chronic inflammation in prostatic carcinogenesis: A 5year followup study. J Urol 2006;176:1012-1016.

42. Hamminga EA, van der Lely AJ, Neumann HA, Thio HB. Chronic inflammation in psoriasis and obesity: Implications for therapy. Med Hypotheses 2006;67:768-773.

43. Datta D, Ferrell WR, Sturrock RD, et al. Inflammatory suppression rapidly attenuates microvascular dysfunction in rheumatoid arthritis. Atherosclerosis 2006;192:391-395.

44. Gallin JI, Snyderman R. Inflammation: Basic Principles and Clinical Correlates. 3rd ed. Philadelphia: Lippincott Williams \& Wilkins, 1999:1.

45. Babior BM, Curnutte JT, Okamura N. The respiratory burst oxidase of the human neutrophils. In: Halliwel B, ed. Oxygen Radicals and Tissue Injury. Proceedings of an Upjohn Symposium. Bethesda, MD: Federation of American Societies for Experimental Biology, 1988:43-48.

46. Kendall EC. The Development of Cortisone as a Therapeutic Agent. Nobel Lecture, December 11, 1950, from Nobel Lectures, Physiology or Medicine 1942-1962, Amsterdam: Elsevier Publishing Company, 1964.

47. Selye H. The evolution of the stress concept. Am Sci 1973;61: 692-699.

48. Mechanism of focal infection. J Am Dent Assoc 1951;42: 619-633.

49. Gammal R. Focal infection references, Medline 1960 to 1998. Online document at: http://health.consumercide.com/ dent-gamm-rt-focal.html Accessed January 2, 2007. 
50. Barnett ML. The oral-systemic disease connection. An update for the practicing dentist. J Am Dent Assoc 2006;137(suppl 2):5S-6S.

51. Mora S, Ridker PM. Justification for the use of statins in primary prevention: an intervention trial evaluating rosuvastatin (JUPITER) - Can C-reactive protein be used to target statin therapy in primary prevention? Am J Cardiol 2006;97(2A): 33A-41A.

52. Grassi D, Lippi C, Necozione S, et al. Short-term administration of dark chocolate is followed by a significant increase in insulin sensitivity and a decrease in blood pressure in healthy persons. Am J Clin Nutr 2005;81:611-614.

53. Kawamori T, Lubet R, Steele VE, et al. Chemopreventive effect of curcumin, a naturally occurring anti-inflammatory agent, during the promotion/progression stages of colon cancer. Cancer Res 1999;59:597-601.

54. Odot J, Albert P, Carlier A, et al. In vitro and in vivo anti-tumoral effect of curcumin against melanoma cells. Int J Cancer 2004;111:381-387.

55. Kurokawa T, Itagaki S, Yamaji T, et al. Antioxidant activity of a novel extract from bamboo grass (AHSS) against ischemia-reperfusion injury in rat small intestine. Biol Pharm Bull 2006;29:2301-2303.

56. Williams ER, Heckman SJ. The local diurnal variation of cloud electrification and the global diurnal variation of negative charge on the earth. J Geophys Res 1993;98:5221-5234

57. Anisimov SV, Mareev EA, Bakastov SS. On the generation and evolution of aeroelectric structures in the surface layer. J Geophys Res 1999;104:14359-14367.

58. Volland H. Atmospheric electrodynamics. In: Lanzerotte LJ, ed: Physics and Chemistry in Space. Berlin: Springer-Verlag, 1984.

59. McMakin CR, Gregoryb WM, Phillips TM. Cytokine changes with microcurrent treatment fibromyalgia associated with cervical spine trauma. J Bodywork Movement Ther 2004;9:169176.

60. Mayor D. Electroacupuncture: A Practical Manual and Resource. Edinburgh: Churchill Livingstone, 2006.

61. Scott-Mumby K. Virtual Medicine. London: Thorsons: HarperCollins, 2004.

62. Malmivuo J, Plonsey R. Bioelectromagnetism. Principles and Applications of Bioelectric and Biomagnetic Fields. New York: Oxford University Press, 1995.

63. Oschman JL. Assume a spherical cow. The role of inflammation in bodywork, energetic and movement therapies. J Bodywork Movement Ther in press.

64. Oschman JL. Our place in nature: Reconnecting with the earth for better sleep. J Altern Complement Med 2004;10:735-736.

65. Huxley TH. Evidence as to Man's Place in Nature. New York: D. Appleton, 1893.

Address reprint requests to:

James L. Oschman, Ph.D.

Nature's Own Research Association

P.O. Box 1935

Dover, NH 03821-1935

E-mail: joschman@aol.com

\section{APPENDIX I}

These cases were selected from randomized controlled clinical studies. Each subject was either provided with an earthing sleep system (ESS) (Fig. 1 in main text) or was connected for 30 minutes to the earthing patch (Fig. 4 in main text) and observed during periodic clinical visits. Progress was monitored with High-Resolution Medical Infrared Imaging and standardized clinical outcome assessment questionnaires (quadruple visual analogue scales for pain severity and quality of sleep).

Case Study \#1. 49-year-old woman. Improvement in circulation and decreased pain after 4 nights sleeping on the ESS. The patient presented on April 11, 2005 complaining of chronic neck and upper back pain, pain interfering with sleep, lack of sleep interfering with daily functioning, leg achiness/restless legs during sleep, and waking stiff and sore. The patient had previously tried a variety of treatments with little improvement. On April 15, 2005 after 4 nights of sleeping on the ESS, the patient reported a $67 \%$ reduction in pain, a $43 \%$ reduction in a lack of sleep interfering with daily functioning, a 29\% reduction in pain interfering with sleep, a 75\% reduction in leg achiness/restless legs during sleep, and an $80 \%$ reduction in waking stiff and sore. The patient reported steady continued improvement on a May 31, 2005 follow-up. Her infrared images documented a pronounced improvement in circulation in the left upper extremity and both lower extremities. The images (Figs. A-1 and A-2) show the significant changes from the baseline images taken on April 11, 2005 (before using the ESS) to the final images taken on April 15, 2005 after 4 nights of sleeping on the ESS.

Case Study \#2. 85-year-old man. Reduction in inflammation that resulted in significant pain resolution after 2 nights sleeping on the ESS. Patient in this study presented on August 9, 2004 with significant chronic left low back pain, chronic recurring right shoulder pain, pain interfering with sleep, and waking stiff and sore over a 4-month period. The patient had been on prolonged medical treatment with little improvement. Subject was provided with an ESS on August 9, 2004 and observed for changes with occasional follow-up clinical visits over an 8-week period. On August 11, 2004, after 2 nights of sleeping on the ESS, the patient reported a 50\% reduction in pain, an $80 \%$ reduction in pain interfering with sleep, and a $75 \%$ reduction in waking stiff and sore. After using the ESS for 4 weeks, the patient reported total resolution of his back and shoulder pain with only occasional mild stiffness. At this time the patient commented, "I have my life back." The images in Figure A-3 document the significant improvement in inflammation in the left lower back region from the baseline images taken on August 9, 2004 (before using the ESS) to the final images taken on August 11, 2004 after 2 nights of sleeping on the ESS. 


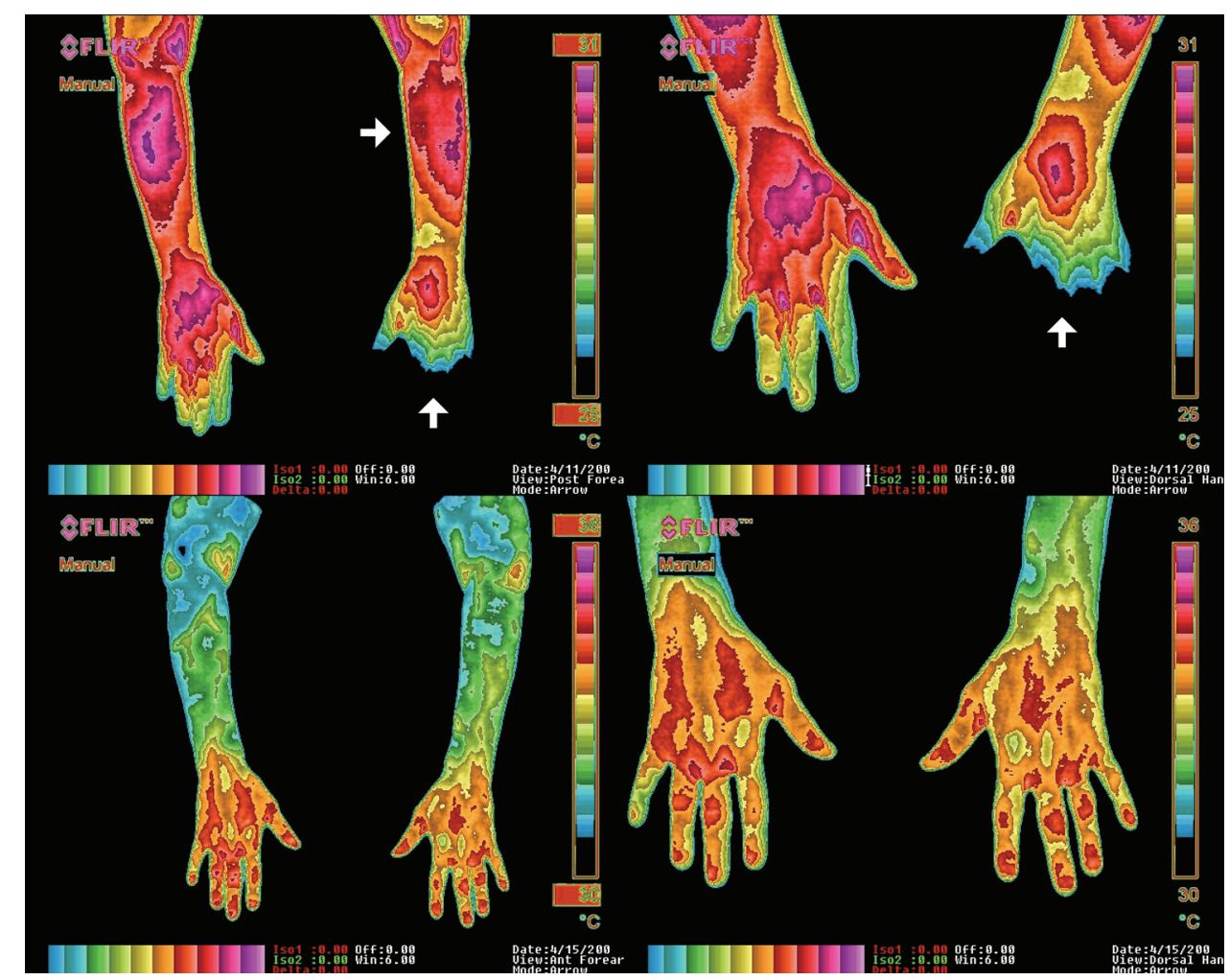

FIG. A-1. Top row images are of the upper extremities taken as a baseline on 4-11-05 (prior to using the earthing sleep system). Arrows denote the areas of poor circulation. The temperature of the left hand is so low that the fingers are at the same temperature as the room and cannot be seen (this is referred to as thermal amputation). The bottom row images were taken on 4-15-05 after 4 nights of sleeping on the earthing sleep system. Note the significant improvement in circulation with a return of normal thermal symmetry in both upper extremities. Temperature scale (color bar) had to be shifted to encompass much warmer thermal profile after earthing. Fingers of left hand were at about 25 degrees $\mathrm{C}$ before earthing and about 34 degrees $\mathrm{C}$ after earthing.

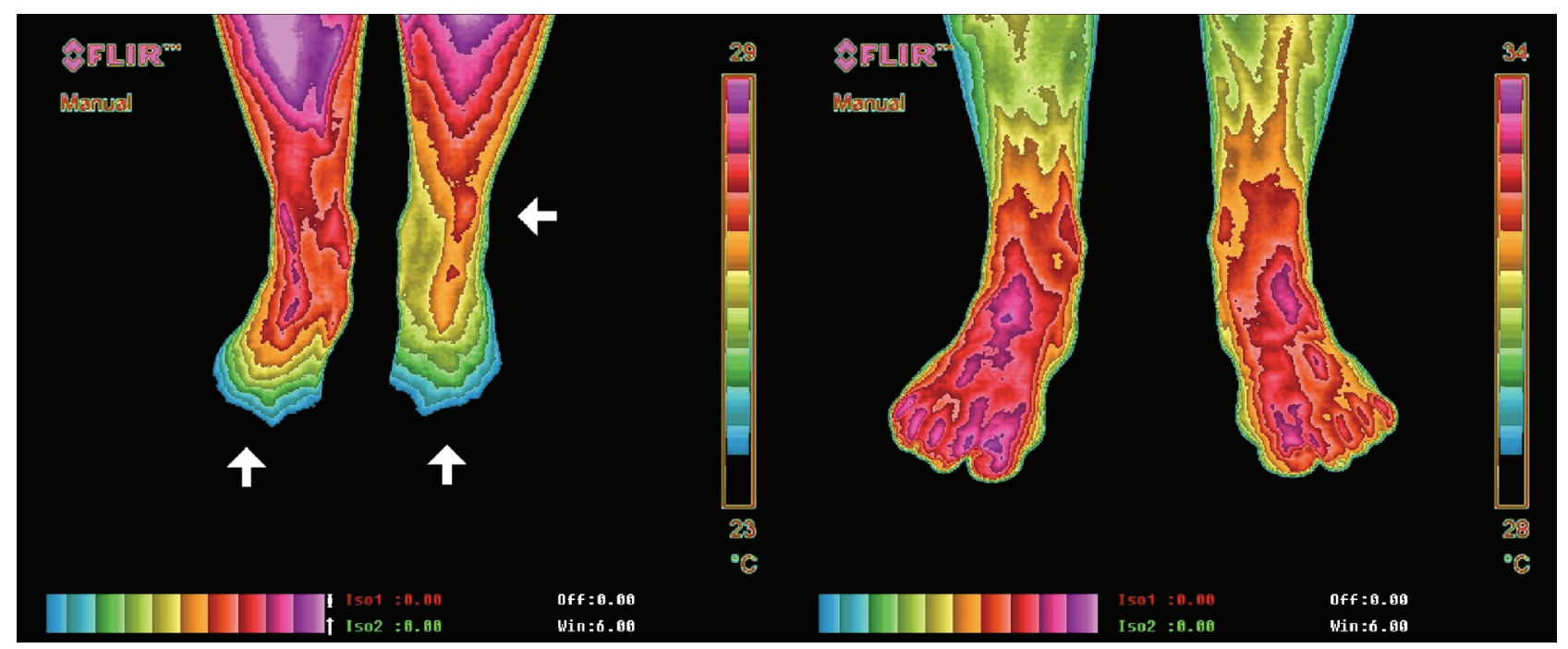

FIG. A-2. Same case study as shown in Figure A-1, showing lower extremities. Left illustration shows baseline on April 11, 2005, prior to use of earthing sleep system. Arrows denote the areas of poor circulation. Temperature of both feet is so low, especially the left, that the toes are at the same temperature as the room and cannot be seen (thermal amputation). Image on right was taken on April 15, 2005, after 4 nights of sleeping on the earthing sleep system. Note significant improvement in circulation with a return of normal thermal symmetry in both feet. Temperature scale (color bar) had to be shifted to encompass much warmer thermal profile after earthing. Before earthing toe temperature was about $24^{\circ} \mathrm{C}$, and after earthing toes were at about $32^{\circ} \mathrm{C}$. 


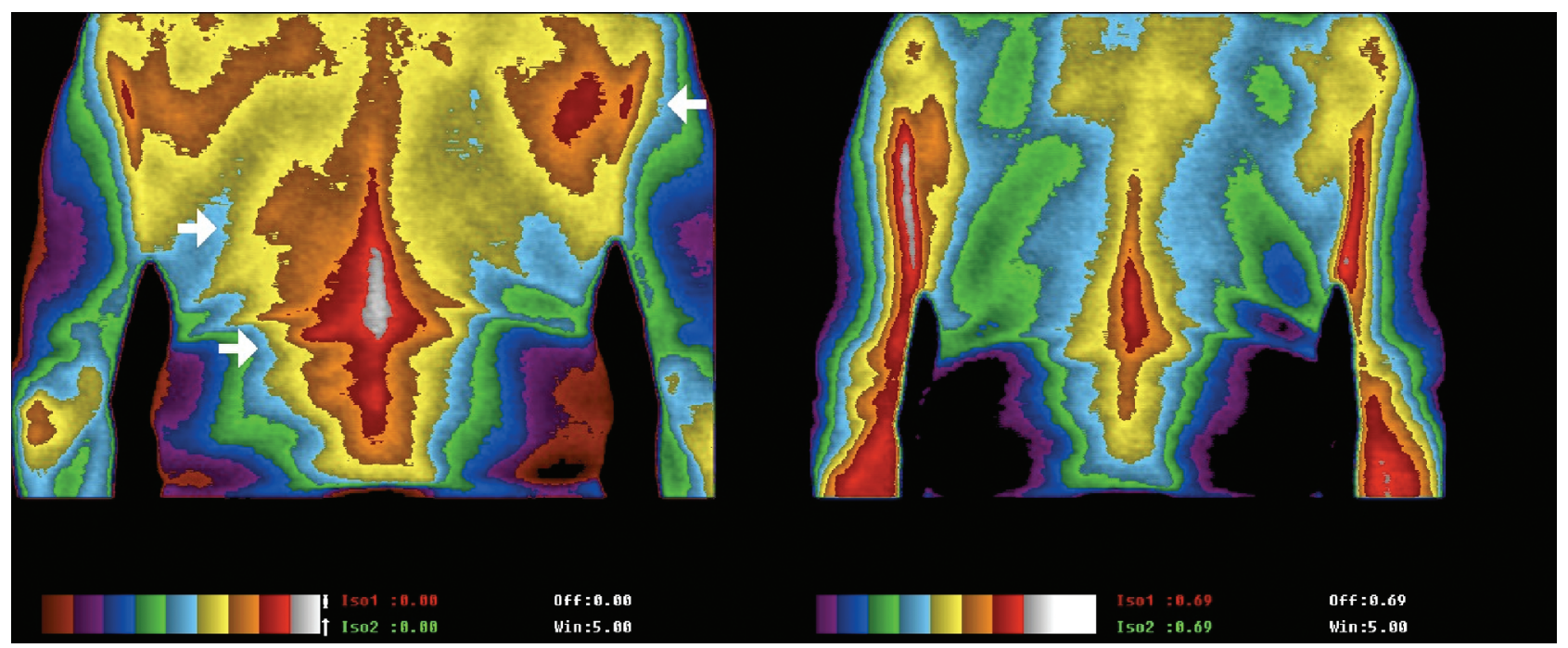

FIG. A-3. Image on left is of the low back taken as a baseline on August 9, 2004 (prior to using earthing sleep system). The arrows denote the most significant areas of inflammation. The image on the right was taken on August 11, 2004 after 2 nights of sleeping on the earthing sleep system. Note the complete resolution of inflammation with a return to normal thermal symmetry. The same color bars were used pre- and postearthing.

Case Study \#3. 48-year-old woman, totally disabled after 6 years of chronic inflammation. Significant reduction in inflammation after 30 minutes exposure to earthing patch system shown in Figure 4 (in main text). This patient presented on September 16, 2004 with significant chronic bilateral knee pain (increased on the right), swollen knees, and fatigue over the past 6 years. Her condition began as a result of an injury sustained while using a ladder. She had received three knee surgeries, prolonged medical treatment, and physical therapy with little improvement. Patient presented with knee wrap supports, a pronounced limp, and ambulated with a walker. Patient was exposed to clinical earthing patch system on September 16, 2004 and observed for changes 30 minutes later and followed up with clinical visits over a 12week period. On September 16, 2004, after 30 minutes of exposure to clinical earthing, the patient reported a $20 \%$ reduction in pain that lasted for 24 hours. After 5 days of clinical earthing, the patient reported a $30 \%$ reduction in pain with an increase in energy that she described as "almost back to my normal self." After 2 weeks of treatment, patient felt good enough to try dancing and reported no increase in symptoms afterward. By 3 weeks, patient no longer needed her walker. After 6 weeks of treatment, patient's limp resolved. At week 12, subject reported an overall 90\% reduction in pain and swelling and noted that, "I can't believe I have my life back." Infrared images (Fig. A-4) show a significant change with a pronounced improvement in inflammation in both knees. The top pre-earthing image is consistent with the patient's statement that her symptoms are worse on the right.

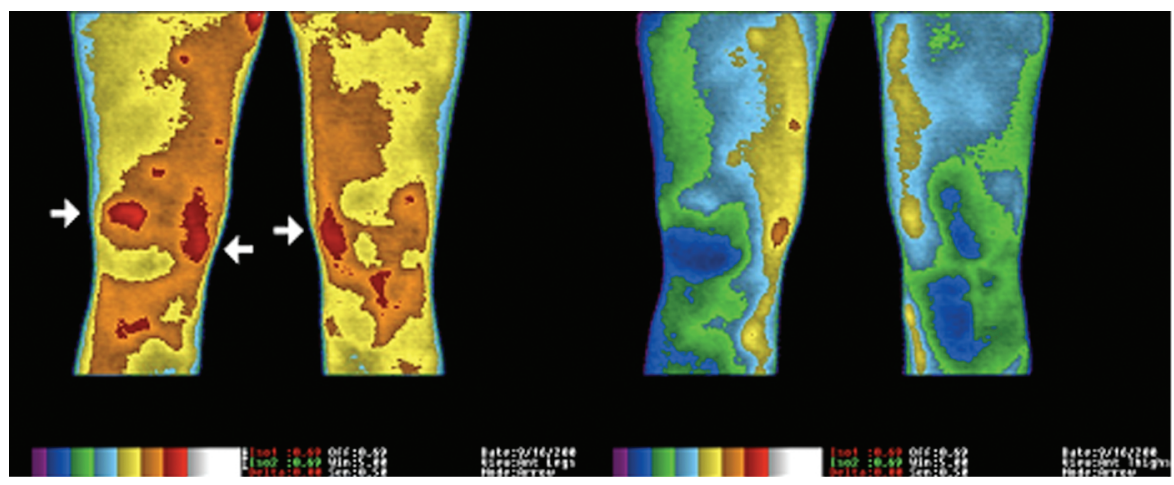

FIG. A-4. Images of the knees taken before and after 30 minutes exposure to clinical earthing on September 16, 2004. The arrows denote the most significant areas of inflammation. Note the considerable reduction in inflammation in the right image taken after 30 minutes of exposure to clinical earthing. Note return of normal thermal symmetry with significant reduction in inflammation. The same color bars were used pre- and postearthing. 